\title{
Symmetry Requirements for 34dB Nonlinearity Compensation in OPC Systems
}

\author{
Mohammad Al-Khateeb ${ }^{(1)^{\star}}$, Mingming Tan ${ }^{(1)}$, Andrew Ellis ${ }^{(1)}$ \\ (1) Aston Institute of Photonic Technologies, Aston University, B4 7ET, Birmingham, UK, \\ alkhamaz@aston.ac.uk
}

Abstract We experimentally achieve compensation of nonlinearity of at least $34 d B$ when deploying Optical Phase Conjugation in an optimized $2^{\text {nd }}$ order distributed Raman system, and demonstrate that the required accuracy for span-to-span power misalignment is below $\pm 1 \mathrm{~dB}$ for $20 \mathrm{~dB}$ compensation.

\section{Introduction}

Several techniques have been proposed to compensate for the nonlinear interference generated in optical fibers ${ }^{1}$. OPC ${ }^{2}$ is one of the most promising techniques as it is an inline alloptical signal processing technique that can act as a transparent compensator for the dispersion and nonlinear interference accumulated in optical fibers for all channels simultaneously, especially when deployed in Distributed Raman Amplified (DRA) links ${ }^{3,4}$. The compensation efficiency is highly dependent on the symmetry of the signal power profile along the system; a full nonlinearity compensation can only be achieved (by OPC) when the signal power evolution is precisely reversed on either side of the OPC. For a $1^{\text {st }}$ order DRA transmission systems ${ }^{5}$; analytical predictions and simulation results have shown that the nonlinearity compensation efficiency can be only enhanced by shortening the Raman pumps spacing regardless of the pumping scheme (backward, forward, or bidirectional). Experimental results ${ }^{5}$ have demonstrated that using OPC-assisted backward pumped $1^{\text {st }}$ order DRA $(2 \times 50 \mathrm{~km})$ achieve a nonlinearity compensation efficiency of $\sim 27 \mathrm{~dB}$. On the other hand, higher order Raman pumping can provide a degree of flexibility in optimising the signal power profile symmetry along the system (for a given Raman pump spacing) ${ }^{6}$. Such systems enable the OPC to achieve maximum nonlinearity compensation efficiency compared to their equivalent of $1^{\text {st }}$ order DRA systems.

In this paper, we experimentally study the optimisation of $2^{\text {nd }}$ order DRA systems showing that they can provide enhanced signal power symmetry, enabling an improved nonlinearity compensation efficiency (at least $\sim 34.5 \mathrm{~dB}$ ) when deploying an OPC between two $50 \mathrm{~km}$ spans, a
$13 \mathrm{~dB}$ improvement compared to $1^{\text {st }}$ order DRA system was observed ${ }^{5}$. Similarly, we show that the nonlinearity compensation efficiency achieved by the OPC is sensitive to the asymmetry in signal power launched into each span along the system, showing that the $2^{\text {nd }}$ order DRA system has a $1.8 \mathrm{~dB}$ power tolerance for a nonlinearity compensation achieved by the OPC of at least $20 \mathrm{~dB}(99 \%)$.

\section{Theoretical Evaluation}

To analytically describe the frequency response of the nonlinear (idler) product generation in DRA systems, we have followed the previously reported method ${ }^{5}$. This method divides each distributed Raman span into $M$ sections over which the gain or loss coefficient is approximately constant. The nonlinearly generated idler from three spectral signal components $\left(E_{q}, E_{r}, E_{s}\right)$ is given by Eq. (1) for a system without OPC and by Eq. (2) for a system with OPC (deployed at midlink). In both equations, $D$ represents degeneracy factor, $N$-number of spans, $L$-span length, $\gamma$-fibre nonlinear coefficient, $\Delta \beta\left(=-4 \pi^{2} \beta_{2}\left[f_{q}-f_{s}\right]\left[f_{r}-f_{s}\right]\right)$ phase mismatching coefficient, $L_{k}$ is the $k^{\text {th }}$ section length, and $g_{k}$ represents exponential gain/loss coefficient for the $k^{\text {th }}$ section. Equations (1\&2) represent a discretised version of the continuous integral ${ }^{7}$ and have been modified to represent the case of different signal powers in each half of the link. Outside the modulus (in Eq.[1] and Eq.[2]), we can see a constant scaling factor and quasi-phase matching term. Inside the modulus we have the coherent summation of the nonlinear optical field generated from each segment in the spans of the first and the second halves of the link. Without an OPC, any misalignment in launched power only results in a variation in the idler power in the strongly phase

$$
\begin{gathered}
P_{F}^{w o / O P C}=\left(\frac{D \gamma}{3}\right)^{2} \frac{\sin ^{2}(N \Delta \beta L / 4)}{\sin ^{2}(\Delta \beta L / 2)}\left|E_{q} E_{r} E_{s}^{*} \sum_{k=1}^{M}\left[\frac{\mathrm{e}^{\left(g_{k}+i \Delta \beta\right) L_{k}}-1}{g_{k}+i \Delta \beta}\right]\left[\prod_{l=1}^{k-1} \mathrm{e}^{\left(g_{l}+i \Delta \beta\right) L_{l}}\right]+E_{q}^{\prime} E_{r}^{\prime} E_{s}^{*} \sum_{k=1}^{M}\left[\frac{\mathrm{e}^{\left(g_{k}+i \Delta \beta\right) L_{k}}-1}{g_{k}+i \Delta \beta}\right]\left[\prod_{l=1}^{k-1} \mathrm{e}^{\left(g_{l}+i \Delta \beta\right) L_{l}}\right]\right|^{2} \\
P_{F}^{w / O P C}=\left(\frac{D \gamma}{3}\right)^{2} \frac{\sin ^{2}(N \Delta \beta L / 4)}{\sin ^{2}(\Delta \beta L / 2)}\left|E_{q} E_{r} E_{s}^{*} e^{(-i \Delta \beta L)} \sum_{k=1}^{M}\left[\frac{\mathrm{e}^{\left(g_{k}+i \Delta \beta\right) L_{k}}-1}{g_{k}+i \Delta \beta}\right]\left[\prod_{l=1}^{k-1} \mathrm{e}^{\left(g_{l}+i \Delta \beta\right) L_{l}}\right]-E_{q}^{* \prime} E_{r}^{* \prime} E_{s}^{\prime} \sum_{k=1}^{M}\left[\frac{\mathrm{e}^{\left(g_{k}-i \Delta \beta\right) L_{k}}-1}{g_{k}-i \Delta \beta}\right]\left[\prod_{l=1}^{k-1} \mathrm{e}^{\left(g_{l}-i \Delta \beta\right) L_{l}}\right]\right|^{2}
\end{gathered}
$$


matched region (i.e. $\Delta \beta \rightarrow 0$ ) due to the change in power itself. On the other hand, with an OPC there may be a significant variation away from (potentially) ideal compensation. Figure1 shows that, for small offsets $( \pm 2 d B)$ the idler power varies by only $3 \mathrm{~dB}$ for a system without an OPC and at least $30 \mathrm{~dB}$ for a system with an OPC.

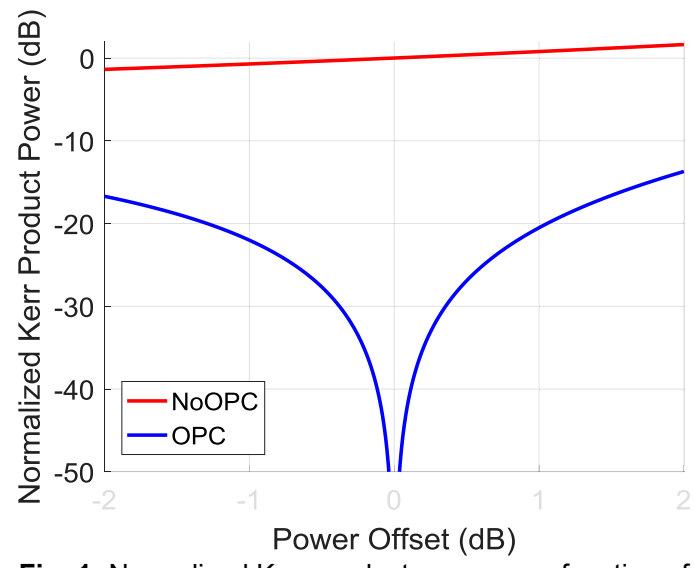

Fig. 1: Normalized Kerr product power as a function of signal launch power misalignment.

\section{Experimental Setup}

Figure 2 shows the experimental set up used to verify Eq. (1) and (2), comprising two $50 \mathrm{~km}$ SSMF spans with second order, 1455nm+1365nm distributed Raman amplification. Idler generation efficiency was established by launching two tuneable Continues Wave (CW) lasers (3dBm each, around 1555nm) and analysing the output with a $150 \mathrm{MHz}$ resolution optical spectrum analyser (OSA). Raman pump powers were optimised to minimise idler generation at $5 \mathrm{GHz}$ with an OPC, but subject to the constraint of $0 \mathrm{~dB}$ net gain, and the inset of Fig. 2 shows an Optical Time-Domain Reflectometer measurement of the power profile and its theoretical fit. The input power to the second span was adjusted using a EDFA, whilst the OPC path had an additional booster amplifier to partially pre-compensate the insertion loss of the OPC $(20 \mathrm{~dB})$. The OPC was a dual band, polarisation insensitive, dual pump OPC ${ }^{8}$. OPCs pumps at $1540.7 \mathrm{~nm}$ and $1570.4 \mathrm{~nm}$ were counter dithered $(60 \mathrm{MHz}+600 \mathrm{MHz})$ to reduce Brillouin scattering in the OPC.

\section{Results and Discussion}

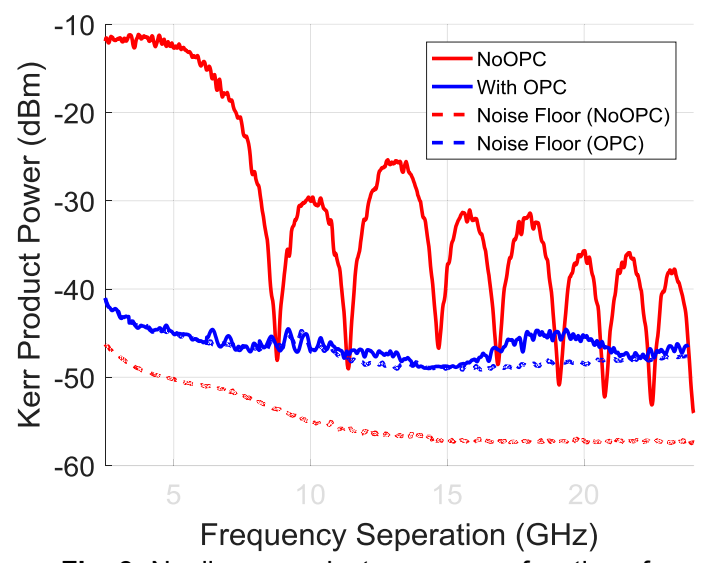

Fig. 3: Nonlinear product power as a function of frequency separation between the two CW lasers.

Figure 3 shows the idler power and the noise floor, with and without OPC, as a function of frequency separation between the two CW lasers. The idler power generated in the system that does not deploy OPC reaches its maximum value $(11.5 \mathrm{dBm})$ at strongly phase matched region and oscillates as a function of frequency separation due phase mismatching accumulation ( $\Delta \beta$ in Eq. [1]). It can be seen that introducing the OPC to the system raised the noise floor by $9 \mathrm{~dB}$, due to the insertion loss of the OPC. On the other

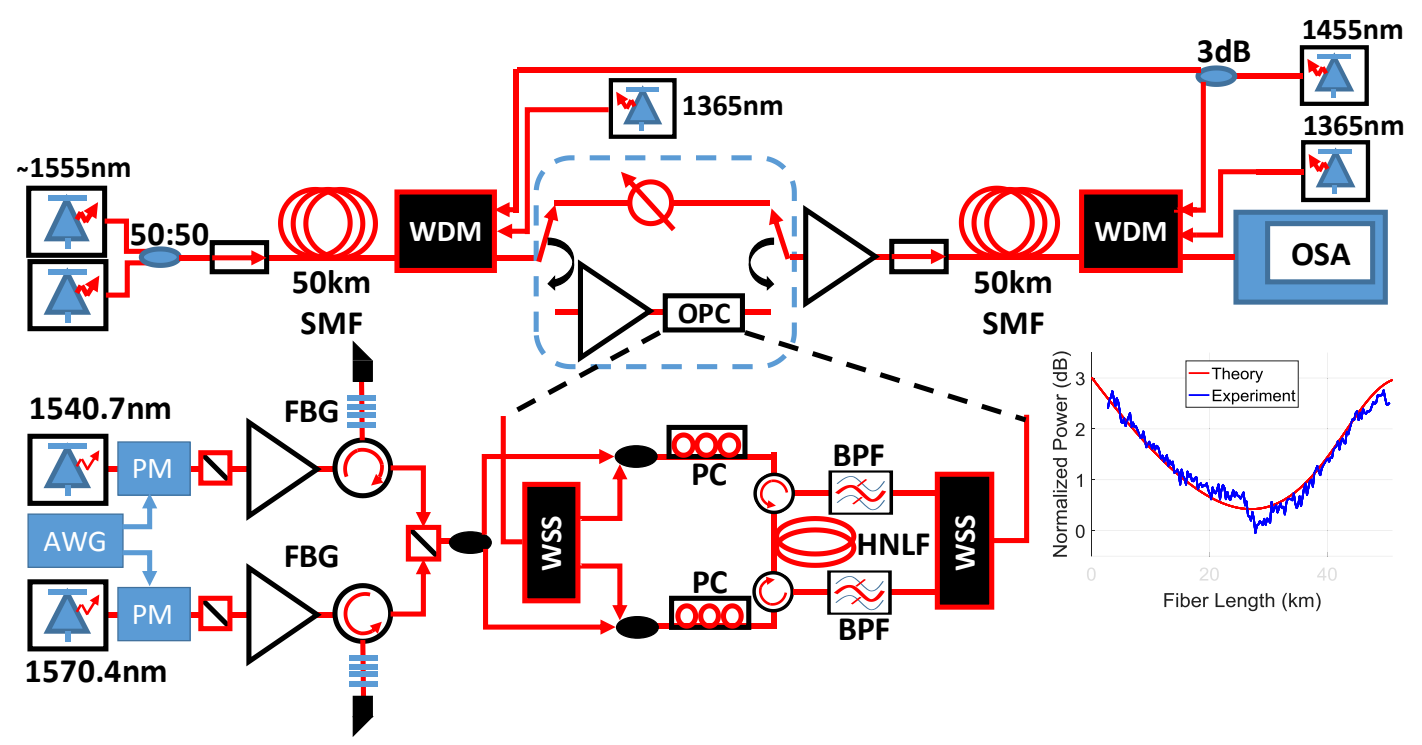

Fig. 2: Experimental setup. 

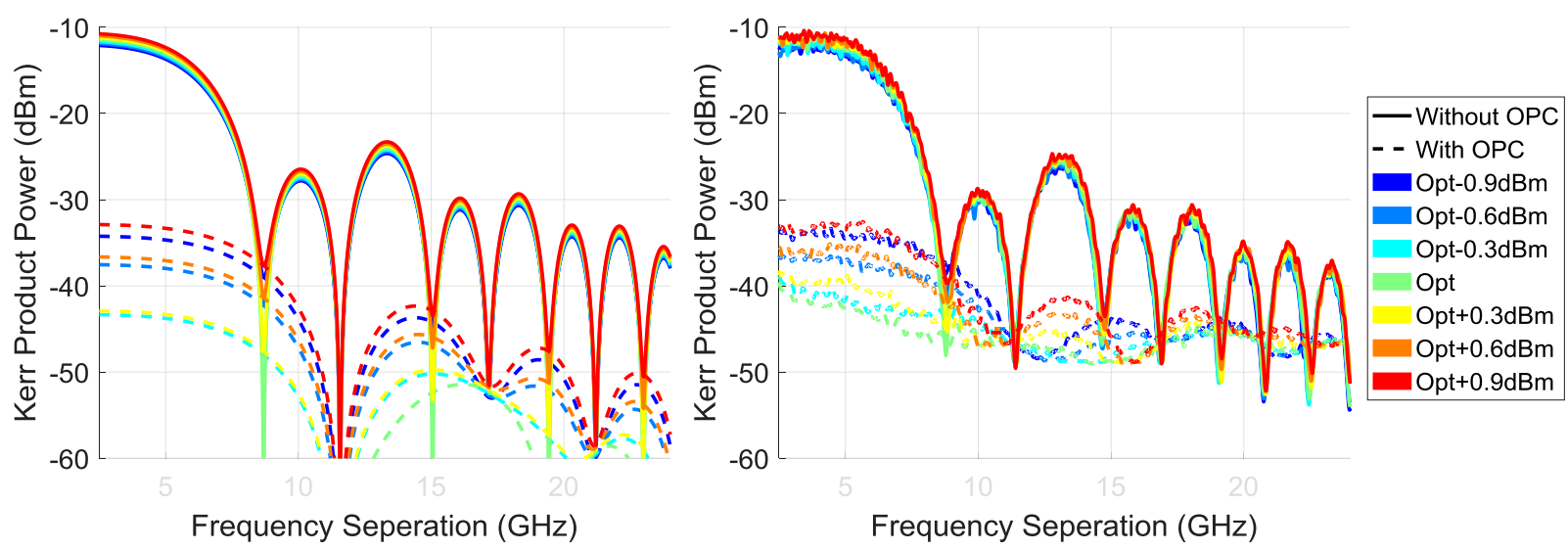

Fig. 4: Nonlinear power as a function of frequency separation calculated theoretically (left), and experimentally measured (right).

hand, we can see that the OPC introduced a significant nonlinearity compensation (at least $34.5 \mathrm{~dB}$ ) with the idler power barely above the noise floor and reached its maximum value at $18 \mathrm{GHz}$ frequency separation. The introduction of $2^{\text {nd }}$ order distributed Raman pumping enabled a $\sim 8 \mathrm{~dB}$ enhancement in nonlinearity compensation when compared to $1^{\text {st }}$ order pumping with the same span length ${ }^{5}$.

Figure 4 shows the effect of the misalignment of the signal power launched into the second span on the idler power for each system both theoretically (left) and experimentally (right). As expected, the launch power misalignment of $\pm 0.9 \mathrm{~dB}$ leads to a strong $(12 \mathrm{~dB})$ degradation in nonlinearity compensation efficiency achieved by the OPC in the strongly phase matched region, and variations in the null positions elsewhere. Without an OPC only minor variations $(\sim 1.5 \mathrm{~dB})$ are observed due to launch power misalignment $(-0.9$ to $0.9 \mathrm{~dB})$ and negligible changes in null position. Taking into account the noise floor, identical trends are observed from the theoretical predictions (calculated from Eq.(1) and Eq.(2), as shown in Fig.4(left)).

\section{Conclusions}

We have experimentally demonstrated the advantage of $2^{\text {nd }}$ order DRA system over $1^{\text {st }}$ order DRA system ${ }^{5}$ in realising high signal power profile symmetry to achieve significant $34 \mathrm{~dB}$ nonlinearity compensation efficiency achieved when deploying mid-link OPC. We also experimentally study, explained by theory, the degradation in nonlinearity compensation efficiency (achieved by OPC) among strongly phase matched signals in the case of misalignment of launch signal power into each half of the link. The results have shown that a $0.9 \mathrm{~dB}$ signal power misalignment can degrade the compensation efficiency by $12 \mathrm{~dB}$.

\section{Acknowledgements}

The work is funded by EPSRC Programme Grant PEACE (EP/L000091/1) and UNLOC (EP/J017582/1). We thank the Dr. Marc Stephens (Aston university) for useful conversations. We thank Sterlite Technologies for providing the transmission fiber, and Finisar for providing a WSS. Original data for this work is available through Aston Research Explorer (https://doi.org/10.17036/researchdata.aston.ac.uk.00 000349).

\section{References}

[1] A. D. Ellis et al., "Performance limits in optical communications due to fiber nonlinearity," Adv. Opt. Photon. 9, 429-503 (2017).

[2] A. Yariv et al., "Compensation for channel dispersion by nonlinear optical phase conjugation," Opt. Lett. 4, 52 (1979).

[3] A. D. Ellis et al., "4 Tb/s Transmission Reach Enhancement Using $10 \times 400 \mathrm{~Gb} / \mathrm{s}$ Super-Channels and Polarization Insensitive Dual Band Optical Phase Conjugation," J. Lightwave Technol. 34, 1717-1723 (2016).

[4] K. Solis-Trapala et al., "Signal power asymmetry tolerance of an optical phase conjugation-based nonlinear compensation system," in The European Conference on Optical Communication (ECOC) (2014), p. 1-3 (We.2.5.4).

[5] M. A. Z. Al-Khateeb et al., "Analysis of the nonlinear Kerr effects in optical transmission systems that deploy optical phase conjugation," Opt. Express 26, 3145-3160 (2018).

[6] P. Rosa et al., "Signal power asymmetry optimisation for optical phase conjugation using Raman amplification," Opt. Express 23, 31772-31778 (2015).

[7] S. Radic et al., "Four-wave mixing in optical links using quasi-distributed optical amplifiers," J. Light. Technol. 19(5), 636-645, (2001).

[8] M. A. Z. Al-Khateeb et al., "Performance Enhancement Prediction for Optical Phase Conjugation in Systems with $100 \mathrm{~km}$ Amplifier Spacing," in Proc. European Conference and Exhibition on Optical Communication (ECOC) (2017), p. Th.1.F.4. 